OPEN ACCESS

Edited by:

Emiliano Cè,

University of Milan, Italy

Reviewed by:

Marta Borrelli,

Università Degli Studi di Milano, Italy

Susanna Rampichini,

University of Milan, Italy

${ }^{*}$ Correspondence:

Jon Ingulf Medbe

jonim@hvl.no

TORCID:

Jon Ingulf Medbe orcid.org/0000-0002-5152-9778

Boye Welde

orcid.org/0000-0003-3805-1615

Specialty section:

This article was submitted to

Exercise Physiology

a section of the journal

Frontiers in Sports and Active Living

Received: 07 September 2021

Accepted: 07 February 2022

Published: 07 March 2022

Citation:

Medbø Jl and Welde B (2022) Effect of Computational Method on

Accumulated $\mathrm{O}_{2}$ Deficit.

Front. Sports Act. Living 4:772049.

doi: 10.3389/fspor.2022.772049

\section{Effect of Computational Method on Accumulated $\mathrm{O}_{2}$ Deficit}

\author{
Jon Ingulf Medb ${ }^{1 * t}$ and Boye Welde ${ }^{2 \dagger}$ \\ ${ }^{1}$ Faculty for Teacher Education, Culture, and Sports, Institute of Language, Literature, Mathematics and Interpretation, \\ Western Norway University of Applied Sciences, Bergen, Norway, ${ }^{2}$ School of Sport Sciences, University of Tromsø The \\ Arctic University of Norway, Tromsø, Norway
}

The aim of this study was to examine how relationships between exercise intensity and the rate of energy release established in different ways, affect the calculated $\mathrm{O}_{2}$ deficit accumulated during strenuous exercise. Aerobic energy release is readily measured by the $\mathrm{O}_{2}$ uptake, while anaerobic energy release is by definition independent of $\mathrm{O}_{2}$. The latter is not easily measured during strenuous exercise, but it can be estimated using the accumulated $\mathrm{O}_{2}$ deficit principle. We have calculated it using nine different approaches. Thirteen moderately trained persons (three women) volunteered to serve as subjects for cycle ergometry. Their maximal $\mathrm{O}_{2}$ uptake was $2.9 \pm 0.6 \mathrm{mmol} \mathrm{s}^{-1}(\bar{x} \pm s ; 3.9 \pm 0.8$ LSTPD $\min ^{-1}$ ). Our reference method (MO) is based on measuring the steady state $\mathrm{O}_{2}$ uptake at the end of at least ten bouts of $10 \mathrm{~min}$ of exercise at constant intensity, varying between 30 and $40 \%$ of that corresponding to the maximal $\mathrm{O}_{2}$ uptake and up to a power $>90 \%$ of the maximal $\mathrm{O}_{2}$ uptake, which is a rather time-consuming method. The outcomes of eight different simpler approaches have been compared with those of the reference method. The main result is that the accumulated $\mathrm{O}_{2}$ deficit calculated depends a great deal on the relationship used to calculate it. A protocol of stepwise increases in exercise intensity every 4 min appeared to work well. A gross efficiency method showed the poorest performance. Another important result is that at constant power the $\mathrm{O}_{2}$ uptake continued to increase beyond 4 min of exercise at all powers examined, also at powers well-below those corresponding to the lactate threshold. Finally, the $\mathrm{O}_{2}$ uptake during loadless pedaling was considerably higher than resting $\mathrm{O}_{2}$ uptake, and it appeared to follow a cubic function of the pedaling frequency. In conclusion, to obtain reliable values of the anaerobic energy release using the accumulated $\mathrm{O}_{2}$ deficit principle, reliable relationships between exercise intensity and $\mathrm{O}_{2}$ demand must be established.

\footnotetext{
Keywords: accumulated $\mathrm{O}_{2}$ deficit, anaerobic energy release, computation method, exercise economy, exercise efficiency, high-intensity exercise, measurement, $\mathrm{O}_{2}$ uptake
}

\section{INTRODUCTION}

Exercising muscles need energy (as ATP) that can be provided by aerobic and anaerobic processes. Aerobic energy release is readily measured by $\mathrm{O}_{2}$ uptake (e.g., Gastin, 2001). Anaerobic energy release is by definition independent of $\mathrm{O}_{2}$ and is not easily measured, for example in relation to strenuous exercise (e.g., Gastin, 1994; Medbø, 2010). Instead, the anaerobic contribution has been estimated by different computational methods. Medbø et al. (1988) proposed using accumulated $\mathrm{O}_{2}$ deficit as an indirect measure of the anaerobic energy 
release during high-intensity exercise. This principle seems to be the only reliable non-invasive measure available (Gastin, 1994). However, the original approach of Medbø et al. (1988) is time consuming and therefore not often used. Several short-cut approaches have been used, but with little validation (Medbø, 2010). In this invited paper we address how eight different (simpler) computational methods affect the calculated anaerobic energy supply in high-intensity activities. We limit our focus to computations involving the accumulated $\mathrm{O}_{2}$ deficit principle.

The main ideas of the accumulated $\mathrm{O}_{2}$ deficit principle are firstly that the rate of energy release (ATP turnover rate) appears to increase linearly with exercise intensity. Thus, if a relationship between exercise intensity and the rate of energy release is established reliably at moderate intensities, it can be extrapolated to higher intensities where anaerobic processes are important (Medbø et al., 1988). A second main idea is that since energy is provided by both aerobic and anaerobic processes, the anaerobic contribution can be taken as the difference between the total energy release and the aerobic part. The aerobic energy release is obtained from the $\mathrm{O}_{2}$ uptake measured during exercise.

The original approach proposed by Medbø et al. (1988) was to measure the $\mathrm{O}_{2}$ uptake at the end of $10 \mathrm{~min}$ of exercise at constant intensity, presumed to be reflect the total rate of energy release (called $\mathrm{O}_{2}$ demand). This must be performed 810 times or more at intensities ranging between 30 and $40 \%$ of the maximal $\mathrm{O}_{2}$ uptake and close to the maximum intensity that can be maintained for $10 \mathrm{~min}$. It would thus require testing over several days.

Several simpler procedures may be proposed. It is widely held that the $\mathrm{O}_{2}$ uptake reaches a steady state value within $3 \mathrm{~min}$ of exercise (e.g., Whipp and Wasserman, 1986; Zoladz et al., 1998), at least for exercise below the lactate threshold (Hill and Lupton, 1923). If so, the duration of each exercise bout may be reduced from 10 to $4 \mathrm{~min}$ or less. Alternatively, exercise may be performed in small stepwise increases of exercise intensity $(<30 \mathrm{~W})$, for example every $4 \mathrm{~min}$. The $\mathrm{O}_{2}$ uptake can be measured at the end of each step. If properly designed, that enables the establishment of the required relationship between exercise intensity and the rate of energy release in $<1 \mathrm{~h}$.

A linear relationship, here between exercise intensity ( $x$; power for cycling) and $\mathrm{O}_{2}$ uptake $(y)$, is described by the intercept and the slope. If the intercept is known, a reliable relationship can be obtained if the $\mathrm{O}_{2}$ demand at one or two intensities is known. The gross efficiency approach sets the intercept to zero. A net efficiency approach can be obtained by either measuring resting $\mathrm{O}_{2}$ uptake or $\mathrm{O}_{2}$ uptake at zero intensity exercise (for example loadless pedaling for cycle exercise) and using that value as the intercept. Medbø et al. (1988) found that while the slope varied considerably between subjects, the intercept did not. They consequently proposed using a common intercept for all subjects taken as the mean of all individual intercepts obtained.

The aim of this study was to examine how different relationships between exercise intensity and the rate of energy release $\left(\mathrm{O}_{2}\right.$ demand) affect the calculated $\mathrm{O}_{2}$ deficit accumulated during strenuous exercise. We have therefore calculated the accumulated $\mathrm{O}_{2}$ deficit using nine different approaches. We have used the original approach proposed by Medbø et al. (1988) as the reference (called method 0, M0) and compared the outcome of eight other possible approaches (M1-M8) with those of the reference.

Our data are from cycling exercise. The principles addressed may be applied to other kinds of exercise too. In the discussion we address two further approaches to estimate the rate of energy release at high exercise intensities with a considerable anaerobic contribution that may be suited for treadmill exercise.

\section{METHODS}

\section{Overview}

Moderately trained subjects repeatedly cycled at constant power for $10 \mathrm{~min}$, and the $\mathrm{O}_{2}$ uptake was measured between 3 and $4 \mathrm{~min}$ of exercise and again at $8-10 \mathrm{~min}$ of exercise. The powers chosen ranged between loadless pedaling and close to the highest power that could be maintained for $10 \mathrm{~min}$. Heart rate and blood lactate concentration were also measured. In further studies the exercise was repeated by using an exercise protocol of 4 min stepwise increases in power. The resting $\mathrm{O}_{2}$ uptake was also measured.

Relationships between power and $\mathrm{O}_{2}$ demand were established in nine different ways using data at different submaximal powers (below those corresponding to the maximal $\mathrm{O}_{2}$ uptake). The relationships obtained were extrapolated to powers corresponding to $100-250 \%$ of those corresponding to the maximal $\mathrm{O}_{2}$ uptake. The accumulated $\mathrm{O}_{2}$ deficit for (simulated) high-intensity exercise was calculated using the nine different relationships obtained.

\section{Subjects}

Thirteen moderately trained volunteers served as subjects. Ten of them were students (three women, seven men), aged $24 \pm 3$ years $(\bar{x} \pm s)$. In addition, three male staff members aged 32,41 , and 55 years also took part in some of the studies. The women were 1.71 $\pm 0.04 \mathrm{~m}$ tall and weighed $74 \pm 7 \mathrm{~kg}$. The men were $1.84 \pm 0.05 \mathrm{~m}$ tall and weighed $82 \pm 7 \mathrm{~kg}$. The subjects' maximal $\mathrm{O}_{2}$ uptake was $2.9 \pm 0.6 \mathrm{mmol} \mathrm{s}^{-1}\left(\bar{x} \pm s ; 3.9 \pm 0.8 \mathrm{~L}_{\mathrm{STPD}} \mathrm{min}^{-1}\right)$.

The subjects were informed both orally and in writing about their rights as volunteers to leave the study at any stage without giving a reason for doing so, before giving their written consent. The study was non-invasive (except for sampling blood from a finger), included only adults capable of consent, and collected only anonymous data. It therefore did not require further ethical approval according to national regulations.

\section{Experiments}

All exercise was performed as cycling on a modified Krogh-type cycle ergometer (Krogh, 1913) at a constant pedaling frequency, which in different experiments was set at between $0.5 \mathrm{~Hz}(30$ $\mathrm{rpm})$ and $2.0 \mathrm{~Hz}(120 \mathrm{rpm})$. Most experiments were carried out at $1.5 \mathrm{~Hz}(90 \mathrm{rpm})$ and again at $0.75 \mathrm{~Hz}(45 \mathrm{rpm})$. The power was varied by changing the braking force. The seat height was adjusted to each subject so that a straight leg reached the pedal without tilting the hip when the pedal was at the lowest position.

The total experimental set-up was quite comprehensive, and all subjects did not take part in all experiments. Twelve subjects performed repeated bouts $(\approx 10-15$ bouts for each 
subject) of 10 min continuous cycling at constant braking force at $1.5 \mathrm{~Hz}$ pedaling frequency. $\mathrm{O}_{2}$ uptake, heart rate and blood lactate concentration were measured at $3-4 \mathrm{~min}$ of exercise and again at $8-10 \mathrm{~min}$. These tests took place over several days; each subject did 4-5 bouts of 10 min duration in each testing session, in increasing order of power. The power was varied between different bouts, ranging from loadless cycling (to allow comparison with resting $\mathrm{O}_{2}$ uptake) to close to a power that could barely be maintained for $10 \mathrm{~min}$, thus on average $95 \%$ of that corresponding to maximal $\mathrm{O}_{2}$ uptake and well-above that corresponding to the lactate threshold. In total, these tests covered the range from loadless cycling to close to a power corresponding to maximal $\mathrm{O}_{2}$ uptake in steps no larger than $22 \mathrm{~W}$. Nine subjects repeated these exercises when cycling at $0.75 \mathrm{~Hz}$ to allow for comparison of effects of different pedaling frequencies.

Nine subjects performed a protocol of stepwise increases in power every 4 min while cycling at $1.5 \mathrm{~Hz} . \mathrm{O}_{2}$ uptake, heart rate and blood lactate concentration were measured near the end of each exercise step. The braking force was increased immediately after each 4 min period with no break or rest. The increase in power from one step to the next was $22 \mathrm{~W}$ for males and $11 \mathrm{~W}$ for females. The power was varied between loadless cycling and a power close to that corresponding to the maximal $\mathrm{O}_{2}$ uptake, thus being well above that corresponding to the lactate threshold. Eight subjects repeated this protocol when cycling at $0.75 \mathrm{~Hz}$.

$\mathrm{O}_{2}$ uptake was measured at rest for ten subjects while the subject sat quietly on the cycle ergometer for $10 \mathrm{~min}$. Nine subjects also cycled at zero load at a pedaling frequency of $0.5 \mathrm{~Hz}$ $(30 \mathrm{rpm})$ to allow comparison with the $\mathrm{O}_{2}$ uptake at rest.

For eleven subjects the maximal $\mathrm{O}_{2}$ uptake was established by the leveling-off criterion of Taylor et al. (1955), modified for ergometer cycling as described by Hermansen (1974). More specifically, the subjects cycled at a high power for $3 \mathrm{~min}$, and the $\mathrm{O}_{2}$ uptake was measured during the last $30-40 \mathrm{~s}$. The test was repeated two to five times for new $3 \mathrm{~min}$ bouts at higher powers with a new measurement of the $\mathrm{O}_{2}$ uptake at the end until the $\mathrm{O}_{2}$ uptake did not increase for a further increase in power. The highest value obtained was taken as the subject's maximal value. It appeared that the highest $\mathrm{O}_{2}$ uptake measured during the repeated $10 \mathrm{~min}$ bouts or the $4 \mathrm{~min}$ stepwise increasing power for these subjects was on average $95 \%$ of the maximum value established by the leveling-off criterion. Two subjects did not carry out the repeated $3 \mathrm{~min}$ bouts. For these two subjects the highest measured $\mathrm{O}_{2}$ uptake measured during the tests was used to indicate their maximum value.

\section{Equipment}

All cycling was done on a Krogh-type cycle ergometer (Krogh, 1913). The ergometer has an instrument recording the pedaling frequency by optical recordings from the sprocket crank wheel. A possible deviation from the preset frequency is shown on an analog instrument. This enables the subjects to keep the pedaling rate constant.

Oxygen uptake was measured by the Douglas bag technique (Douglas, 1911). Expired air was collected in Douglas bags while the sampling time was recorded. After collection the closed bag was transferred to a bench and emptied, while the sampled volume was measured with an S430-A ventilation measuring system with a K520-C521 flow transducer (Applied Electrochemistry, Pittsburgh, PA, USA) while the air temperature was measured simultaneously by a digital thermometer. During the emptying a small sample of expired air was extracted to measure fractions of $\mathrm{O}_{2}$ and $\mathrm{CO}_{2}$ with instruments of Applied Electrochemistry; $\mathrm{O}_{2}$ : an $\mathrm{S} 3 \mathrm{~A} / \mathrm{I}$ analyzer with an $\mathrm{N}-$ $22 \mathrm{M}$ zirconium oxide-type $\mathrm{O}_{2}$ sensor; $\mathrm{CO}_{2}$ : a $\mathrm{CD}-3 \mathrm{~A}$ analyzer with a P-61B infrared-type $\mathrm{CO}_{2}$ sensor). The instruments were calibrated by high-quality gases of known concentrations, and by a 7 L syringe (series 4900, Hans Rudolph, Kansas City, MO, USA). Heart rate was measured by a PE 3000 heart rate recorder (Polar Electro OY, Kempele, Finland). Blood lactate concentration was measured from blood taken from a cleaned finger by the LT-1710 Lactate Pro ${ }^{\mathrm{TM}}$ analyzer (Arkray Factory Inc., KDK Corporation, Shiga, Japan). A separate examination has shown that this instrument measures blood lactate concentration quite accurately, with a random error of $0.5 \mathrm{mmol} \mathrm{L}^{-1}$ or less (Medbø et al., 2000). The lactate threshold was taken as the exercise intensity corresponding to a blood lactate concentration of 4.0 $\mathrm{mmol} \mathrm{L}{ }^{-1}$, as established by a linear interpolation if necessary. This is a simple, straightforward criterion that has been shown to provide a value close to the maximal lactate steady state criterion (Mamen et al., 2011).

\section{Computational Methods Examined}

We examined eight different methods for establishing the relationship between power and $\mathrm{O}_{2}$ demand as alternatives to the one proposed by Medbø et al. (1988; called M0 here). For all models (described below) it is assumed that $\mathrm{O}_{2}$ demand increases linearly with exercise intensity that for cycling is taken as the power. The first four methods tested (M1-M4 below) use around ten pairs of values for each subject to estimate both the slope and the intercept (not M2) of the relationships in question.

M0 Method 0, proposed by Medbø et al. (1988), was used as the reference method. It is based on repeated measurements of the steady state $\mathrm{O}_{2}$ uptake for at least 8-10 different bouts at constant power of $10 \mathrm{~min}$ duration. The powers used varied between 30 and $40 \%$ and $>90 \%$ of that corresponding to the maximal $\mathrm{O}_{2}$ uptake. There are non-linear effects at low powers (see Figure 1 for an example), and measurements below the non-linear threshold have not been included. The regression coefficients were found by ordinary least square regression.

M1 Method 1 uses data gathered as for method 0, but the $\mathrm{O}_{2}$ uptake was measured between 3 and 4 min of exercise during a $10 \mathrm{~min}$ bout. The only difference between methods 0 and 1 is the duration of cycling before a measurement is taken.

M2 Method 2 uses the same data as method 1, but in addition the $y$-intercept is a forced value (the mean of the intercepts of method $1 ; 0.50 \mathrm{mmol} \mathrm{s}^{-1}$ for the present data). The only difference between methods 1 and 2 is the forced $y$-intercept of method 2 .

M3 In method 3 exercise starts at a rather low power, $<100 \mathrm{~W}$. Between 3 and $4 \mathrm{~min}$ of exercise, $\mathrm{O}_{2}$ uptake was measured. 

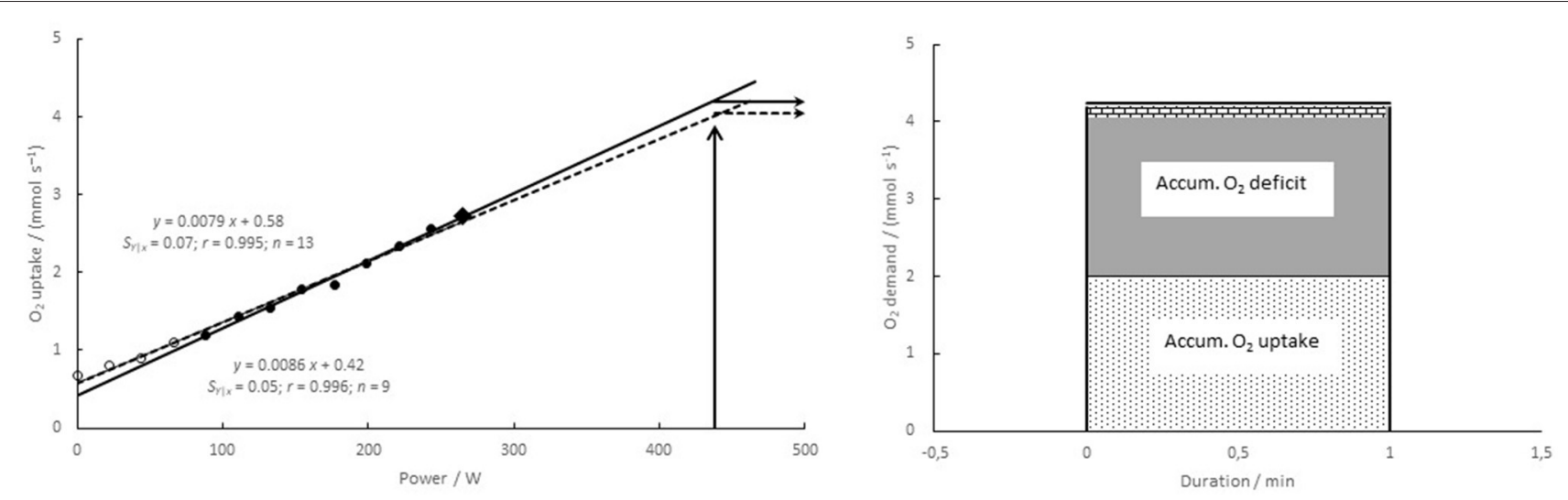

FIGURE 1 | (Left) relationship between power and $\mathrm{O}_{2}$ demand for a typical subject (no. 8). $\mathrm{O}_{2}$ uptake was measured at 8-10 min of exercise at nine different constant powers between 88 and $265 \mathrm{~W}$ (filled symbols), and a linear regression was calculated based on those data. The highest value (filled diamond) was used in methods M5-M8 (see Figure 2 below). If in addition four measurements at lower powers are included (open symbols), a different relationship with a higher value of the intercept and a lower slope is obtained (dashed line, called method 4). (Right) calculations of the accumulated $\mathrm{O}_{2}$ deficit. $\mathrm{O}_{2}$ uptake was measured during 1 min exercise to exhaustion (here taken from a previous study), giving the accumulated $\mathrm{O}_{2}$ uptake (light dotted area). The accumulated $\mathrm{O}_{2}$ demand is taken as the $\mathrm{O}_{2}$ demand times the duration, reflecting the total energy release in units of oxygen. The accumulated $\mathrm{O}_{2}$ deficit (dark gray area + hatched area at the top) is taken as the accumulated $\mathrm{O}_{2}$ demand minus the accumulated $\mathrm{O}_{2}$ uptake. If the $\mathrm{O}_{2}$ demand is estimated to be lower (here by method 4), the estimated accumulated $\mathrm{O}_{2}$ deficit becomes lower (hatched area at the top not included).
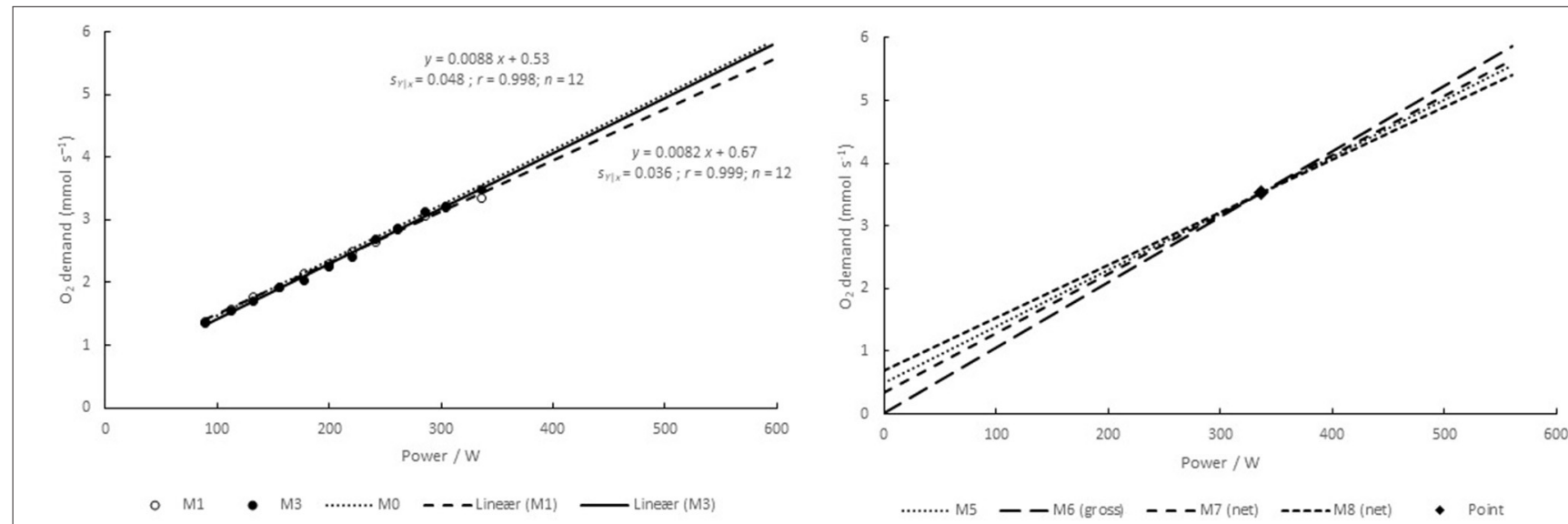

FIGURE 2 | Relationship between power and $\mathrm{O}_{2}$ demand for a typical subject (no. 9), and the effects of extrapolations to high powers. (Left) relationships established by methods 1 and 3 (M1 and M3). The relationship of M2 is very close to that of M1, and it is therefore not included. The relationship of the reference method (M0) is shown by the dotted line. A corresponding relationship for M4 is shown in Figure 1. (Right) relationships established by methods 5-8 (M5-M8) using fixed intercepts and one measurement at around $90 \%$ of the maximal $\mathrm{O}_{2}$ uptake (point). The line of $\mathrm{M} 5$ is very close to that of $\mathrm{MO}$, which is therefore not included.

Immediately after the measurement the intensity was raised by a small step (11-22 W, depending on the subject's physical level; no break or rest between steps), and a new 4 min cycle was repeated. This procedure was repeated at least ten times, giving more than ten paired measurements of the $\mathrm{O}_{2}$ uptake vs. power in $<1 \mathrm{~h}$. The regression coefficients were determined using least square regression.

M4 As shown in Figure 1, there are non-linear effects at low powers. Including measurements at low power will affect the calculated relationships between power and $\mathrm{O}_{2}$ uptake. Method 4 includes all measurements, even those at loadless pedaling. The regression coefficients were calculated by least square regression.
The last four methods use a fixed intercept and only one or two individual measurements. The slope is found as follows: The $\mathrm{O}_{2}$ uptake is measured near the end of a $10 \mathrm{~min}$ bout at a power corresponding to around $90 \%$ of the maximal $\mathrm{O}_{2}$ uptake. A linear relationship is taken from the intercept (presumed $\mathrm{O}_{2}$ uptake at zero power) through the measured $\mathrm{O}_{2}$ uptake and the corresponding power (Figure 2).

M5 For method 5 the mean of the individual intercepts (see methods 0 and 2) was calculated and used as a common intercept for all subjects. $\mathrm{O}_{2}$ uptake is measured at a power corresponding to $\geq 90 \%$ of maximal $\mathrm{O}_{2}$ uptake (e.g., diamond in Figure 1), and a linear relationship is taken from the intercept through the measured value. 
M6 Method 6, the gross efficiency method, sets the intercept to zero. The $\mathrm{O}_{2}$ uptake at a high power $\left(\geq 90 \%\right.$ of maximal $\mathrm{O}_{2}$ uptake) is measured, and a linear relationship is taken from the zero intercept through this measured value.

M7 Method 7 is a net efficiency method. The $\mathrm{O}_{2}$ uptake is first measured at rest, giving the intercept, and then at a power corresponding to $\geq 90 \%$ of the maximal $\mathrm{O}_{2}$ uptake. A linear relationship is taken from the intercept through this latter measured value.

M8 Method 8 is also a net efficiency method. The $\mathrm{O}_{2}$ uptake is first measured during loadless cycling, giving the intercept, and then at a power corresponding to $\geq 90 \%$ of the maximal $\mathrm{O}_{2}$ uptake. A linear relationship is taken from the intercept through this latter measured value.

Method 0 has been used in some studies (Medbø et al., 1988; Medbø and Tabata, 1989, 1993; Tabata et al., 1996, 1997; Buck and McNaughton, 1999). Method 1 has been used by Green and Dawson (1996) and further examined by Buck and McNaughton (1999). Method 2 has been recommended by Noordhof et al. (2010, 2013). Method 3 has been used by Duffield et al. (2007). Bosquet et al. (2007a,b) and Riojas et al. (2020) used a similar protocol with 2-min steps, thus increasing the power well before the $\mathrm{O}_{2}$ uptake reaches a (near) steady state. Method 3 has also been recommended by Medbø et al. (1988) and by Medbø (2010). While Medbø et al. (1988) warned against possible flaws when using method 4, they recommended method 5 as an alternative to the proposed reference method. Method 5 has later been used in many studies (Scott et al., 1991; Ramsbottom et al., 1994, 1997, 2001; Maxwell and Nimmo, 1996; Doherty, 1998; Wadley and le Rossignol, 1998; Doherty et al., 2000, 2002; Russell et al., 2000; Bickham et al., 2002; Nevill et al., 2008). The gross efficiency principle (method 6) has been recommended by many (e.g., Ettema and Lorås, 2009; Noordhof et al., 2013) and more recently used for cross-country skiing (Andersson and McGawley, 2018). The two net efficiency methods (no. 7 and 8) do not seem to have been greatly used.

\section{Calculations and Modeling}

For high intensity exercise, anaerobic energy release can be estimated by the accumulated $\mathrm{O}_{2}$ deficit, assuming that the $\mathrm{O}_{2}$ uptake measured at the end of the $10 \mathrm{~min}$ bouts described above is equal to the total rate of energy release in those exercises. The established linear relationship between exercise intensity and the rate of energy release is also assumed to hold for higher intensities where there is a considerable anaerobic contribution. The $\mathrm{O}_{2}$ uptake is measured throughout the whole high intensity exercise, giving the accumulated $\mathrm{O}_{2}$ uptake as the aerobic contribution. The anaerobic part, called the accumulated $\mathrm{O}_{2}$ deficit, is taken as the difference between the total energy release and the measured aerobic part (Figure 1 above); see Medbø et al. (1988 or 2010) for further details. This means that different relationships between exercise intensity and energy release will give different values for the accumulated $\mathrm{O}_{2}$ deficit calculated, as shown in Figure 1.

Exercise at very high intensity leads to exhaustion in a few seconds. Exercise may be maintained for a longer duration if the load is lighter. In our modeling we assume exercise at constant intensity to exhaustion. The present study does not include experiments at powers above that corresponding to the maximal $\mathrm{O}_{2}$ uptake. To allow extrapolations to supramaximal intensities we have used data from our former studies (Table 1). More specifically, the data of Medbø and Tabata (1989) and Medbø et al. (1999) suggest that to cause exhaustion in 10 and $30 \mathrm{~s}$ typical subjects must cycle at a power corresponding to $\approx 250$ and $200 \%$, respectively, of their maximal $\mathrm{O}_{2}$ uptake. The data of Medbø and Tabata (1989) suggest that the average subject can exercise at an intensity $\approx 150$ and $120 \%$ of that corresponding to the maximal $\mathrm{O}_{2}$ uptake, for 1 and $2 \mathrm{~min}$, respectively, before exhaustion is reached. By an extrapolation beyond their measurements, their data also suggest that exercise at a power corresponding to $100 \%$ of the maximal $\mathrm{O}_{2}$ uptake will exhaust the subject in about $3.6 \mathrm{~min}$ (exactly $217 \mathrm{~s}$ ). This latter estimate is at variance with experience from laboratory experiments. We have nevertheless used this value as an estimate for further modeling, which we address again in the discussion.

Medbø (2010) pooled data from 30 different studies on the relative contribution of $\mathrm{O}_{2}$ taken up during exercise to the total energy release. Exercise duration ranged from about $10 \mathrm{~s}$ to more than $5 \mathrm{~min}$, and all exercises were performed to exhaustion. The model produced enables estimation of the accumulated $\mathrm{O}_{2}$ uptake as a proportion of the total energy release as a function of the time to exhaustion (see Figure 8 in Medbø, 2010), and these estimates are summarized in Table 1. Consequently, when estimating the total rate of energy release by our reference method, the accumulated $\mathrm{O}_{2}$ uptake is estimated to be $75 \%$ of the total for the $3.6 \mathrm{~min}$ exercise to exhaustion, and $10 \%$ of the total for the $10 \mathrm{~s}$ sprint at $250 \%$ of the maximal $\mathrm{O}_{2}$ uptake (Table 1).

To apply these values to our data for further calculations, we used average values of our 13 subjects, for example the

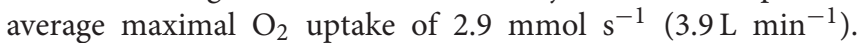
Therefore, an exercise intensity of 120 and $250 \%$ of the maximal $\mathrm{O}_{2}$ uptake corresponds to an $\mathrm{O}_{2}$ demand of 3.5 and $7.3 \mathrm{mmol}$ $\mathrm{s}^{-1}$, respectively.

The total rate of energy release $(Y)$ is assumed to be related to the power $(P)$ by the linear relationship

$$
Y=a+b P
$$

where $a$ and $b$ are, respectively, the intercept and slope of the linear relationships. This equation is readily solved for the power, giving

$$
P=\frac{Y-a}{b}
$$

The power for each exercise model in Table 1 was calculated using the regression coefficients obtained for the reference method (M0 above). Thus, exercise at 120 and 250\%, for example, corresponds to a power of 343 and $768 \mathrm{~W}$, respectively, for the average subject (Table 1).

For linear relationships obtained by different protocols we have assumed the same power, time to exhaustion, and accumulated $\mathrm{O}_{2}$ uptake. The estimated $\mathrm{O}_{2}$ demand and thus the (estimated) total energy release will differ depending on the linear 
TABLE 1 | Assumptions for the calculations and modeling.

\begin{tabular}{|c|c|c|c|c|}
\hline $\begin{array}{l}\text { Exercise intensity/ } \\
\text { (percent of max. } \mathrm{O}_{2} \text { uptake) }\end{array}$ & $\begin{array}{l}\text { Assumed time to } \\
\text { exhaustion }\end{array}$ & $\begin{array}{c}\text { Accumulated } \mathrm{O}_{2} \text { uptake/ } \\
\text { (percent of total energy release) }\end{array}$ & $\begin{array}{l}\mathrm{O}_{2} \text { demand/ } \\
\left(\mathrm{mmol} \mathrm{s}^{-1}\right)\end{array}$ & $\begin{array}{l}\text { Estimated } \\
\text { power/W }\end{array}$ \\
\hline 100 & $3.62 \min (217 \mathrm{~s})$ & 75 & 2.9 & 274 \\
\hline 120 & $2 \min (120 s)$ & 63 & 3.5 & 343 \\
\hline 150 & $1 \min (60 s)$ & 47 & 4.4 & 438 \\
\hline 200 & $30 s$ & 30 & 5.8 & 603 \\
\hline 250 & $10 \mathrm{~s}$ & 10 & 7.3 & 768 \\
\hline
\end{tabular}

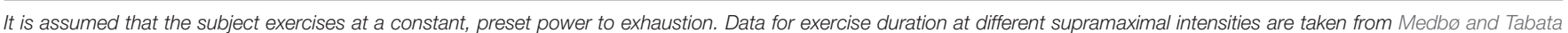

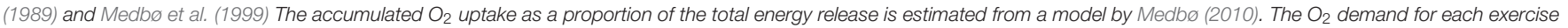

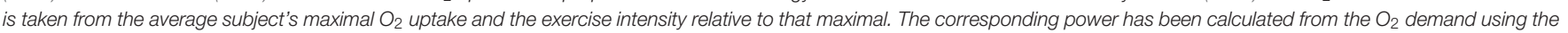
mean of the regression parameters of the subjects. See the main text for further details.

parameters used, as will the accumulated $\mathrm{O}_{2}$ deficit calculated by the different models.

All calculations were performed on a spreadsheet (Excel version 2021).

\section{Statistics}

Results are summarized as mean $\pm s(\bar{x})$ (standard error of the mean). Possible changes were examined by Gosset's ("Student's") one-sample $t$-test for matched-pair measurements on the same subject, and further by a two-way analysis of variance (IBM SPSS version 27), using Dunnett's post-hoc test (M0 as the reference). Linear regressions were calculated using the builtin regression tools in the spreadsheet (in the package for data analysis). Non-linear curve fits (cubic and square) were calculated by using the option of polynomial regression in the plotting menus.

Not all subjects carried out all experiments. When some data are missing from a subject, the statistical analysis used automatically excludes all data for that subject for the test in question. The effect is a somewhat lower test power, while no systematic effect is introduced.

\section{RESULTS}

\section{$\mathrm{O}_{2}$ Uptake vs. Exercise Protocol $1.5 \mathrm{~Hz}$ Cycling}

$\mathrm{O}_{2}$ uptake was measured between 3 and 4 min of exercise and again between 8 and $10 \mathrm{~min}$ of cycling for $10 \mathrm{~min}$ at constant power (between 70 and $\approx 300 \mathrm{~W}$ ) at $1.50 \mathrm{~Hz}$. There was a small but statistically significant increase in the $\mathrm{O}_{2}$ uptake of 0.044 $\pm 0.005 \mathrm{mmol} \mathrm{s}^{-1}$ during the last $6 \mathrm{~min}$ of exercise $(n=155$ paired measurements on 12 subjects; $+2.7 \%$; $P<0.001$ ). For measurements below the individual lactate threshold the increase was $0.026 \pm 0.004 \mathrm{mmol} \mathrm{s}^{-1}(n=126$ measurements; $+1.8 \% ; P$ $<0.001$ ), while for measurements at or above the threshold the increase was $0.113 \pm 0.012 \mathrm{mmol} \mathrm{s}^{-1}$ ( $n=33$ values; $+4.9 \%, P$ $<0.001)$. The blood lactate concentration measured just after the highest power maintained for $10 \mathrm{~min}$ was $8.9 \pm 1.8 \mathrm{mmol} \mathrm{L}^{-1}(\bar{x}$ $\pm s)$.

Nine subjects performed cycling at stepwise increasing power every 4 min. $\mathrm{O}_{2}$ uptake was measured during 3-4 min of exercise at each step. $\mathrm{O}_{2}$ uptake was $0.015 \pm 0.007 \mathrm{mmol} \mathrm{s}^{-1}$ higher during $4 \mathrm{~min}$ stepwise increasing power than after $4 \mathrm{~min}$ of cycling with rest before a new exercise $(P=0.03) . \mathrm{O}_{2}$ uptake was $0.026 \pm 0.007 \mathrm{mmol} \mathrm{s}^{-1}$ lower during $4 \mathrm{~min}$ stepwise increasing power than after $10 \mathrm{~min}$ of cycling at the same power with rest before the exercise $(P<0.001)$.

\section{$0.75 \mathrm{~Hz}$ Cycling}

Nine subjects cycled at $0.75 \mathrm{~Hz}$ for $10 \mathrm{~min}$ at different constant powers where the $\mathrm{O}_{2}$ uptake was measured at 3-4 and 8$10 \mathrm{~min}$ of cycling. The findings were similar to those at $1.50 \mathrm{~Hz}$. In brief, the $\mathrm{O}_{2}$ uptake increased with time both for cycling below $(+2.1 \% ; n=109$ paired comparisons; $P<$ $0.001)$ and at or above the lactate threshold $(+5.1 \% ; n=19$; $P<0.001)$.

Eight of these subjects performed the protocol of $4 \mathrm{~min}$ stepwise increasing power at $0.75 \mathrm{~Hz}$ frequency. $\mathrm{O}_{2}$ uptake was measured during 3-4 min of exercise at each step and compared with the values obtained at the same power after 4 and $10 \mathrm{~min}$ of cycling with a rest before a new exercise bout. For 106 paired comparisons the values of the two $4 \mathrm{~min}$ protocols did not differ $\left(-0.002 \pm 0.007 \mathrm{mmol} \mathrm{s}^{-1}\right.$ for the stepwise protocol vs. after $4 \mathrm{~min}$ of $10 \mathrm{~min}$ bouts). The $\mathrm{O}_{2}$ uptake was $0.033 \pm$ $0.006 \mathrm{mmol} \mathrm{s}^{-1}$ lower during $4 \mathrm{~min}$ stepwise increasing power than after $10 \mathrm{~min}$ of cycling with rest before each exercise $(P<0.001)$.

\section{$\mathrm{O}_{2}$ Uptake at Low Powers}

$\mathrm{O}_{2}$ uptake was measured during loadless pedaling and for increasing powers up to close to the maximum that could be maintained for $10 \mathrm{~min}$. For powers below about $75 \mathrm{~W}(\approx 1 \mathrm{~W}$ $\mathrm{kg}^{-1}$ body mass) the measured $\mathrm{O}_{2}$ uptake was somewhat higher than that expected based on linear extrapolation of relationships established at higher powers (see Figure 1 above for a typical example). Consequently, measurements at powers $<75 \mathrm{~W}$ were not included (except for method 4). For cycling at $0.75 \mathrm{~Hz}$ the non-linear effect was less pronounced. At that frequency the lower limit was set to $30 \mathrm{~W}$. Including measurements below the proposed lower limit resulted in relationships with a higher intercept but a lower slope, as addressed further below (see results for method 4). 

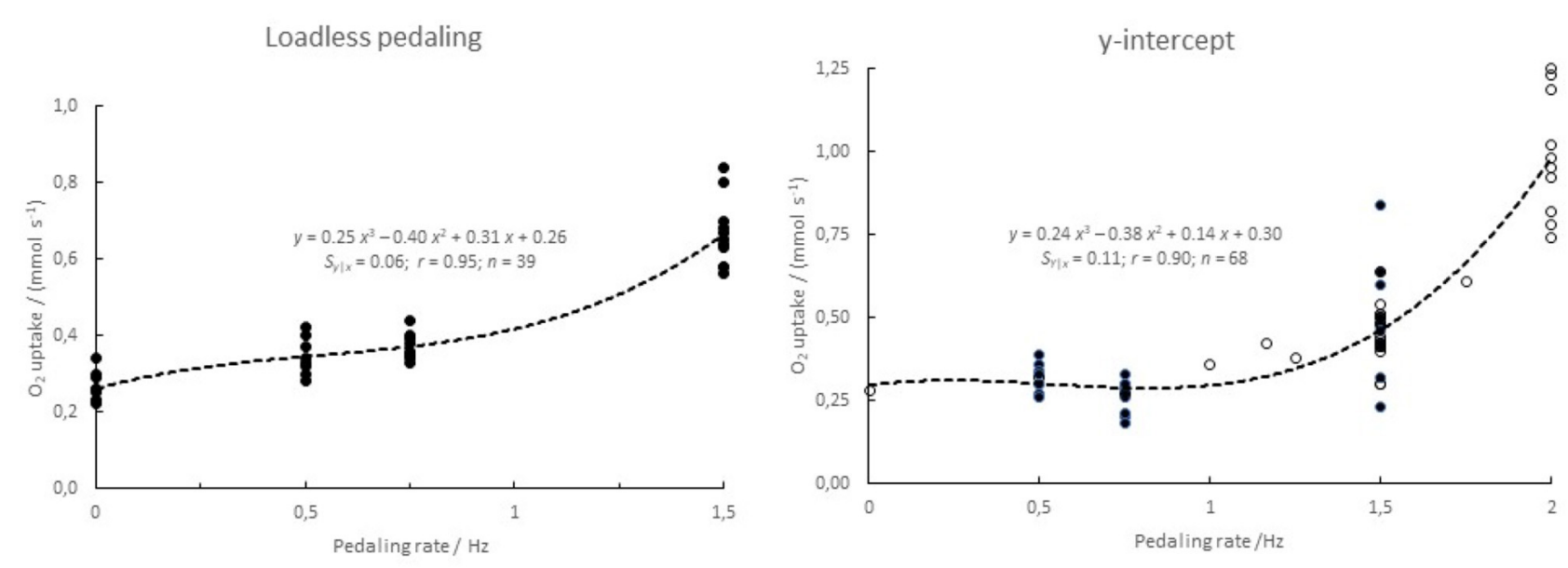

FIGURE $3 \mid \mathrm{O}_{2}$ uptake as a function of pedaling frequency. (Left) loadless pedaling of $n=8-11$ subjects. (Right) y-intercept of linear regressions of steady-state $\mathrm{O}_{2}$ uptake on power. The data for the latter curve are taken from this study $(0.5,0.75$ and $1.5 \mathrm{~Hz} ; n=8,10$, and 12 , respectively, filled symbols) and from Medbø and Tabata (1989) and Medbø et al. (1999; open symbols).

\section{Intercept and $\mathrm{O}_{2}$ Uptake vs. Pedaling Frequency}

$\mathrm{O}_{2}$ uptake was measured at rest while the subject sat still on the cycle ergometer and further during loadless pedaling at $0.5,0.75$, and $1.5 \mathrm{~Hz}$ cycling frequency. The data obtained are compatible with a cubic (also a square) relationship between the frequency and the $\mathrm{O}_{2}$ uptake (Figure 3, left panel). The y-intercept was established at different pedaling rates. The data are compatible with the idea that the $y$-intercepts follow a cubic (also square, not shown) relationship with the pedaling frequency (Figure 3, right panel).

\section{Effect of Relationship Between Power and $\mathrm{O}_{2}$ Uptake on Estimated Accumulated $\mathrm{O}_{2}$ Deficit}

Both the intercepts and the slopes differed systematically between the different methods $(P<0.001)$. More specifically, Dunnett's post-hoc test revealed different intercepts of methods M4, M6, M7, and M8 vs. M0. There was no significant difference in the intercepts of M1 or M3 vs. that of M0 (and obviously no difference for M2 and M5 using mean values as intercepts). Likewise, the slopes of M1, M4, M6, M7, and M8 differed significantly from that of M0, while the slopes of M2, M3, and M5 did not. Consequently, the relationships also differed systematically when extrapolated to intensities above that corresponding to the maximal $\mathrm{O}_{2}$ uptake, which is shown in Figures 1, 2 above.

The accumulated $\mathrm{O}_{2}$ deficit for five different exercise intensities to exhaustion was calculated by the nine different methods as explained above. The values differ significantly between the models used $(P<0.001)$ as well as between subjects and exercise durations $(P<0.001)$. The results are summarized in Table 2.

\section{Methods Based on Many Individual Data}

The results obtained when using $\mathrm{O}_{2}$ uptakes measured after 34 min of exercise instead of 10 min bouts (methods 1 and 2) are systematically lower than those of the reference method $(P<$ 0.001 ), in particular for the longest exercise durations where the values were around $15 \%$ lower than those of the reference method (Figure 4, upper left panel). The main reason for the lower estimates is the lower slope coefficient. Using a fixed, common y-intercept (method 2) apparently reduced the difference to the values of the reference method (Table 2). The main reason is a number of outliers from one subject (see Figure 4, upper right panel).

The results of the $4 \mathrm{~min}$ stepwise increases in power (method 3) are on average very close to those of the reference method for all five exercise combinations examined. Method 4 examined the effect of including all measurements at the end of $10 \mathrm{~min}$ bouts for calculating the regression parameters, even those at low powers where non-linear effects were seen. In this case the calculated values were systematically lower (around 6\%) than those obtained by the reference method $(P<0.001)$.

\section{Methods Assuming a Fixed, Common Intercept and Few Individual Values}

Method 5 is based on a common intercept taken as the mean of all subjects. The slope was taken assuming a linear relationship from that intercept through the point corresponding to the highest power that was maintained for $10 \mathrm{~min}$, and the corresponding $\mathrm{O}_{2}$ uptake. The slope of this approach was similar to that of the reference method, and consequently the accumulated $\mathrm{O}_{2}$ deficit calculated by this method was on average only $\approx 1 \%$ larger than the values of the reference method (Figure 5).

The gross efficiency method (no. 6) has a zero intercept and a slope that was nearly $13 \%$ higher than that of the reference method. This method gave values $\geq 15 \%$ larger than those of 
TABLE 2 | Intercept and slope of nine different approaches examined, and the accumulated $\mathrm{O}_{2}$ deficit calculated for each approach for five different exercise conditions.

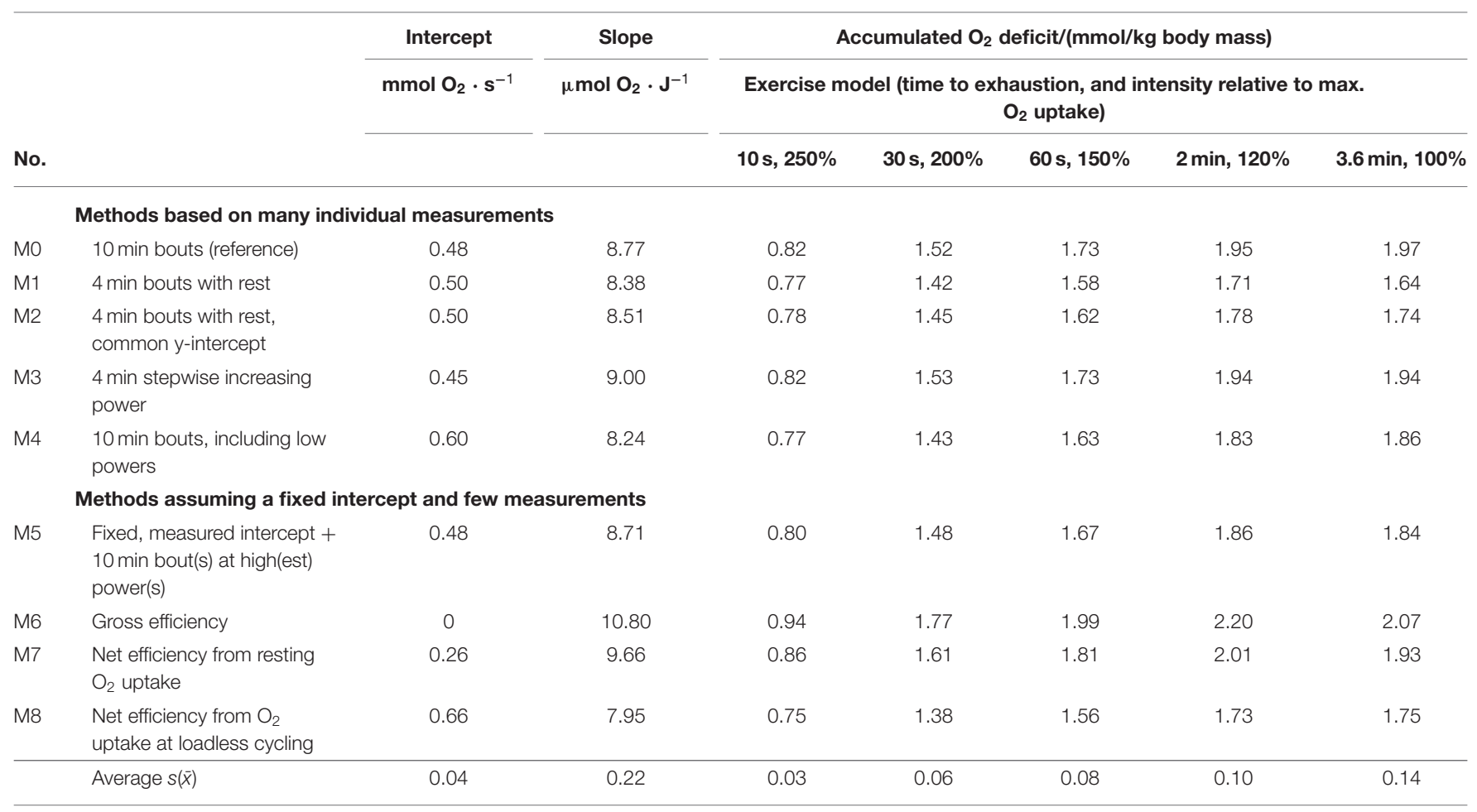

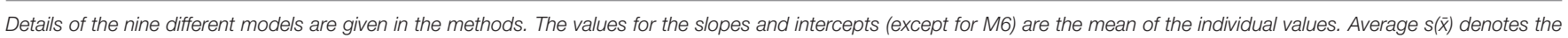
standard error of the mean, averaged across the nine methods.

the reference method $(P<0.001)$. This method also showed the largest between-subjects variability.

Two versions of the net efficiency method were examined. For the one assuming a linear relationship from resting $\mathrm{O}_{2}$ uptake at zero load (no. 7), the intercept was lower and the slope was 9\% higher than that of the reference method. The accumulated $\mathrm{O}_{2}$ deficit calculated by this method was on average $\approx 5 \%$ higher than the corresponding values of the reference method $(P<$ 0.001). For method number 8 the net efficiency was calculated using the $\mathrm{O}_{2}$ uptake measured during loadless pedaling as the intercept. The slope of this method was $8 \%$ lower than that of the reference method, and consequently the accumulated $\mathrm{O}_{2}$ deficit calculated by this method was on average $\approx 10 \%$ lower than the corresponding values obtained by the reference method $(P<0.001)$.

\section{Variations Between Subjects}

Figures 4, 5 show that there was considerable variability between subjects in performing the tested methods vs. the reference method. Both the average bias (addressed above) and the between-subjects variations differed systematically between the methods (Table 3). The between-subjects variations were least for methods 1 and 4 , also quite small for method 3 that appeared unbiased, and largest for method 6 (gross efficiency). Moreover, the methods using a fixed y-intercept (M2 and M5M8) showed in general larger variability than those establishing the intercept individually (M1, M3, and M4). In particular, methods 1 and 2 used the same data except that for method
2 a common y-intercept was used. Data of one outlying subject appeared to reduce the bias vs. the reference method but increase the between-subjects variation for method 2 compared with method 1.

\section{DISCUSSION}

The main result of this study is that the calculated value of the accumulated $\mathrm{O}_{2}$ deficit depends a great deal on the relationship used to calculate the value. The relationship changes if $\mathrm{O}_{2}$ at low exercise intensity is included. Further, the relationships are also influenced by the pedaling frequency.

\section{Methodological Considerations}

We used data from repeated bouts of 10 min duration. The reason for that choice is that the $\mathrm{O}_{2}$ uptake did not reach a steady state within $3 \mathrm{~min}$ of exercise, even if the exercise intensity was well below that corresponding to the lactate threshold. Data from our previous study suggest that there is little change beyond $10 \mathrm{~min}$ of exercise (Medbø and Tabata, 1993). It is widely held that below the lactate threshold the $\mathrm{O}_{2}$ uptake reaches a steady state value within $3 \mathrm{~min}$ of exercise at constant intensity (e.g., Whipp and Wasserman, 1986; Zoladz et al., 1998). Our data, based on more than one hundred paired comparisons, suggest that that view needs to be revised.

Method 0, based on repeated bouts of $10 \mathrm{~min}$ duration, is our reference method. It has been argued that because of the slow component in the $\mathrm{O}_{2}$ uptake by time at exercise intensities above 


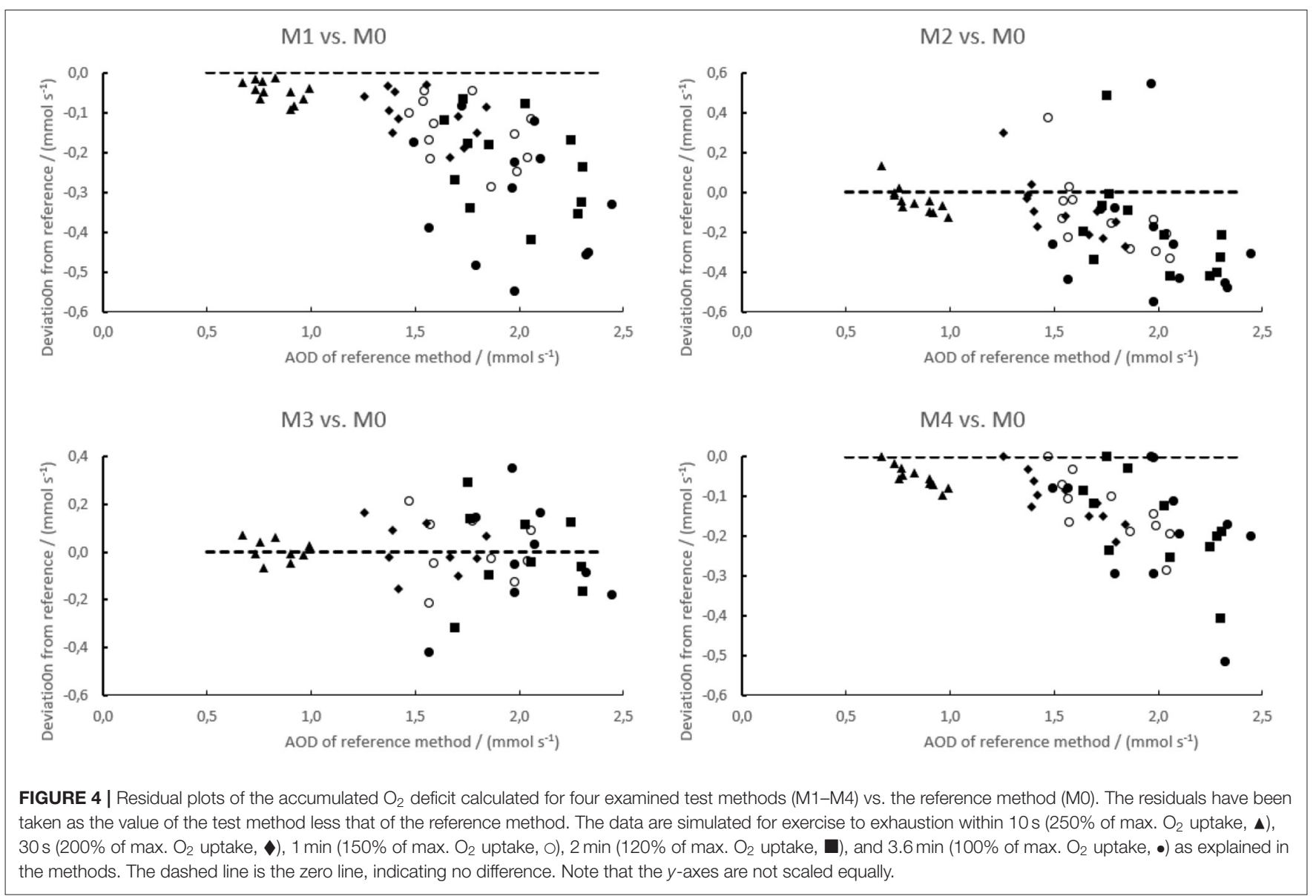

the lactate threshold, the reference method will overestimate the anaerobic energy release. Consequently, methods 1 or 2 might be more reliable (e.g., Noordhof et al., 2010, 2013). However, Tabata et al. (1997) found good agreement between the accumulated $\mathrm{O}_{2}$ deficit obtained during 4 min intermittent cycling at $170 \%$ of the maximal $\mathrm{O}_{2}$ uptake, and the maximal accumulated $\mathrm{O}_{2}$ deficit established by the reference method, when cycling continuously at $\approx 120 \%$ of the maximal $\mathrm{O}_{2}$ uptake to exhaustion. That would not be the case if the two quantities had been calculated using methods 1 or 2. Likewise, data by Buck and McNaughton (1999) suggest that the accumulated $\mathrm{O}_{2}$ deficit calculated by our reference method provides reliable data, while method 1 underestimates the value considerably.

We have calculated the accumulated $\mathrm{O}_{2}$ deficit from modeled data with no direct measurements of high-intensity exercise. One consequence of the modeling was that exercise at an intensity of no more than $100 \%$ of that corresponding to the maximal $\mathrm{O}_{2}$ uptake apparently leads to exhaustion in $217 \mathrm{~s}$ ( $3.6 \mathrm{~min})$. This is clearly in conflict with common experience. However, we use these data only for comparisons of the outcome of different calculations. The effect of a misestimate will affect the different computational methods in a similar way, and thus not affect the outcome of the comparisons. These considerations suggest on the other hand that better models may be needed in future studies.
Our data in Figure 3 suggest that the $\mathrm{O}_{2}$ uptake during loadless cycling and the intercepts of the relationships of $\mathrm{O}_{2}$ uptake vs. power can be described by a cubic function of the pedaling frequency. That is compatible with a response in proportion to the power for accelerating the legs (see e.g., Formenti et al., 2015). However, while the leg during (loaded) cycling is accelerated during the first part of a push downwards, thus gaining kinetic energy, it is decelerated during the later part of the push, transferring its kinetic energy to the ergometer, as also addressed by Ettema and Lorås (2009). Corresponding considerations hold for kinetic energy added to the leg during a (passive) lift during the upstroke. These considerations suggest that other factors than accelerations and decelerations of the legs may cause the excess $\mathrm{O}_{2}$ uptake seen at high pedaling rates. There may for example be compensatory use of muscles in the upper body, muscle work that may not be regained as mechanical work on the ergometer. It is not known to what extent the observed cubic relationships really reflect a causal or mechanistic relationship. In line with that, second-order polynomials fit the data almost as well as the cubic ones used (not shown). The data nevertheless support the idea that at high speeds effects related to accelerations and decelerations may be important.

We measured $\mathrm{O}_{2}$ uptake with the Douglas bag technique and measured volume and fractions of $\mathrm{O}_{2}$ and $\mathrm{CO}_{2}$ in inspired and 


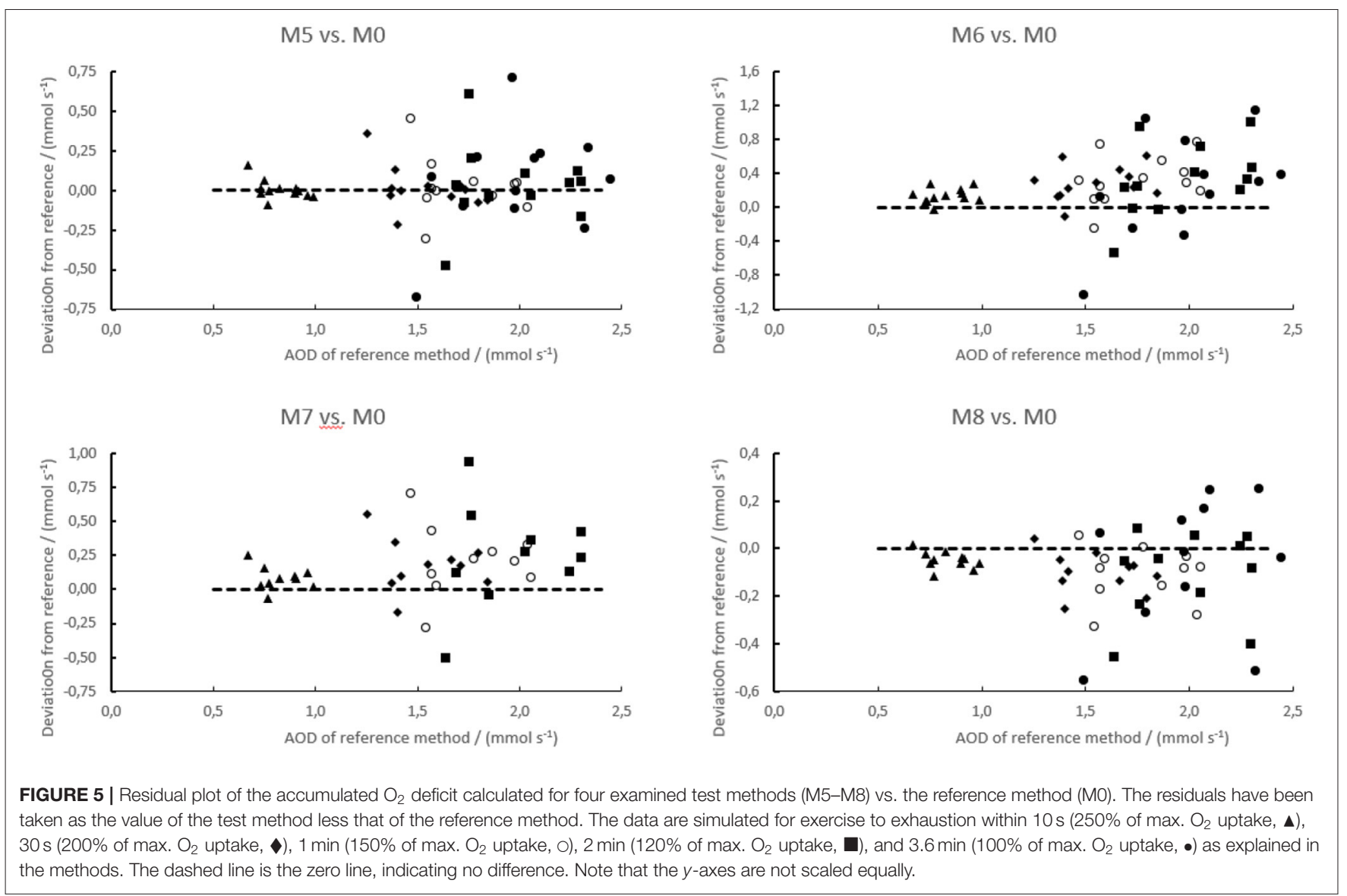

expired air using high-quality instruments. This allowed us full control of the outcome, including how the data (e.g., $\mathrm{O}_{2}$ uptake) are calculated from the measured values. The performance of automatic systems may on the other hand be influenced by built-in corrections (Medbø, 2002, 2010).

\section{Evaluation of the Different Calculation Models Methods Using Many Individual Values}

Method 1, repeated bouts of 4 min duration separated by a few minutes of rest, gave significantly lower values than those of the reference method. The reason is the drift in the $\mathrm{O}_{2}$ uptake beyond $4 \mathrm{~min}$ of exercise. Therefore, the slope calculated from data after $4 \mathrm{~min}$ of exercise was less than for the reference method, leading to a calculated accumulated $\mathrm{O}_{2}$ deficit 6-15\% below the values of the reference method. Method 2 performed similarly to method 1 except for data for one subject. We do not recommend the use of these short-cut methods, at least unless it can be shown that our reference method overestimates the $\mathrm{O}_{2}$ demand.

Method 3 with its small steps of increases in exercise intensity every 4 minute is a commonly used protocol for testing. The whole test can be carried out in one session, usually lasting $<1 \mathrm{~h}$. The relationship between power and $\mathrm{O}_{2}$ demand established by this method was similar to that of the reference method, and consequently the accumulated $\mathrm{O}_{2}$ deficit calculated by this method did not differ greatly from that of the reference method. Moreover, since the protocol used is similar to that of finding the lactate threshold, the two tests may be combined. This fast method may serve as a suitable alternative to the reference method. That conclusion is in line with an earlier study on treadmill running (Medbø et al., 1988).

There are non-linear effects at low exercise intensity. For treadmill running, these may be associated with disproportional vertical body movements at low running speeds (see also Medbø et al., 1988). For cycling, they may be related to movements of the legs (repeated accelerations and decelerations) and compensatory use of muscles in the upper body, as addressed above. The effect is more pronounced at high than at low pedaling rates, but it was also present in cycling at only $0.5 \mathrm{~Hz}$. As an extreme measure, we included all measurements below our proposed lower threshold of inclusion in our method 4 . The slopes obtained were lower than those of the reference method, but the intercepts were higher. The accumulated $\mathrm{O}_{2}$ deficit calculated by this method was $5-6 \%$ less than those of the reference method. The mismatch is clearly less if values only slightly below the non-linear threshold are included. Thus, inclusion of a few values slightly below the recommended lower limit does not seem to have much effect. In the results we have only shown this effect for bouts of $10 \mathrm{~min}$ duration. There is also a corresponding effect for methods 1-3. 
TABLE 3 | Between-subjects variations in differences in accumulated $\mathrm{O}_{2}$ deficit calculated by the eight methods examined vs. the reference method.

\begin{tabular}{|c|c|c|c|c|c|}
\hline Method & 10 s, $250 \%$ & 30 s, $200 \%$ & $1 \mathrm{~min}, 150 \%$ & $2 \min , 121 \%$ & $3.6 \mathrm{~min}, 100 \%$ \\
\hline M1 & 0.03 & 0.06 & 0.08 & 0.11 & 0.15 \\
\hline M2 & 0.07 & 0.15 & 0.19 & 0.25 & 0.29 \\
\hline M3 & 0.05 & 0.11 & 0.14 & 0.18 & 0.23 \\
\hline M4 & 0.03 & 0.06 & 0.08 & 0.12 & 0.15 \\
\hline M5 & 0.06 & 0.14 & 0.18 & 0.25 & 0.33 \\
\hline M6 & 0.09 & 0.20 & 0.28 & 0.43 & 0.61 \\
\hline M7 & 0.09 & 0.19 & 0.26 & 0.38 & 0.50 \\
\hline M8 & 0.04 & 0.08 & 0.12 & 0.18 & 0.28 \\
\hline
\end{tabular}

M1-M8 refer to methods 1-8. The values are the standard deviations for 9-12 subjects and expressed in $\mathrm{mmol}_{2} \mathrm{~s}^{-1}$.

\section{Methods Assuming a Fixed, Common Intercept and Few Individual Values}

While the slope of $\mathrm{O}_{2}$ uptake vs. treadmill speed varies systematically between subjects, the intercept does not vary much (Medbø et al., 1988), see also the outcomes of methods 1 and 2 in this study. This may suggest using one common intercept for all subjects. However, the intercept varies systematically between exercise conditions, as shown in Figure 3 for different pedaling frequencies. This means that a value found for one exercise condition cannot be used for a different condition, as also pointed out earlier (Medbø and Tabata, 1989; Medbø et al., 1999).

Method 5 used a common, fixed intercept taken as the mean for all subjects in the study. That was the recommended alternative to the reference method proposed by Medbø et al. (1988), and that approach has also been recommended by Bickham et al. (2002). That method also worked quite well in this study since the intercepts did not vary greatly between subjects despite considerable variations in the slopes. As pointed out above, the intercept must be established for each exercise condition, which may limit the use of this method.

Method 6 used the gross economy or efficiency principle. Its intercept is zero. The slope was consequently too high, as were the values of the accumulated $\mathrm{O}_{2}$ deficit calculated by this method. The outcome of this method also showed the largest between-subjects variability. We do not recommend the use of this method, at least not for cycling and running. As pointed out by Boone and Bourgois (2012), delta efficiency rather than gross efficiency seems to be the best measure of exercise economy.

Method 7 used the net economy or efficiency principle, using resting $\mathrm{O}_{2}$ uptake as the intercept. That value of the intercept was clearly too low at only around half the value of the intercepts established by the measured data. The slope established by this method was therefore too high as were the values of the accumulated $\mathrm{O}_{2}$ deficit. We do not recommend use of this method, at least not for cycling and running.

Method 8 also used the net economy or efficiency principle, but for this method the intercept was taken as the $\mathrm{O}_{2}$ uptake during zero load cycling. That value is too high because of the non-linear effects seen at low exercise intensities addressed above. The slope was therefore lower than that of the reference method, as was the accumulated $\mathrm{O}_{2}$ deficit calculated by this method. We do not recommend use of this method, at least not for cycling and running.

We used only one measurement of the steady state $\mathrm{O}_{2}$ uptake for methods 5-8. Medbø et al. (1988) recommended using 23 measurements as a safeguard since erroneous measurements occasionally occur. In this study we saw only a few poor measurements among around a thousand taken, and we could check those used in the calculations with other values as needed. If that means of validation is not available, we recommend 2-3 measurements rather than just one, as proposed earlier by Medbø et al. (1988).

The use of the gross efficiency principle has been strongly recommended by e.g., Ettema and Lorås (2009), and it has more recently been used by e.g., Andersson and McGawley (2018) to test sprint skiing. If the method is based on measurements of the steady state $\mathrm{O}_{2}$ uptake at high powers, it is numerically robust. That holds for all methods using a fixed intercept and few measurements (e.g., methods 58 in this study). The zero intercept of method 6 is clearly misleading. Methods 5, 7, and 8 use intercepts that are more realistic. These methods are simple to carry out once the intercept has been established. Consequently, we consider the gross efficiency method to be inferior to any of these alternative methods.

\section{Suggestions for Further Studies and Development}

A basic assumption of the accumulated $\mathrm{O}_{2}$ deficit principle is that a linear extrapolation is justified. As pointed out above, at high speeds acceleration of body segments may be a significant part of the work done by the muscles. Since the kinetic energy increases as the square of the speed, there may be significant non-linear effects at high speeds, in particular if the frequency also increases. In line with that, Andersson and McGawley (2018) suggest that non-linear effects may be significant during roller skiing on the treadmill. These facts may suggest nonlinear curve fits, but non-linear fits are numerically non-robust and therefore not suited for extrapolations. We here address 
some possibilities that may deserve further examination. If correctly implemented, the $\mathrm{O}_{2}$ demand may be established at the exercise intensity in question, thus eliminating the need for an extrapolation.

\section{Short Interval Principle}

During short interval training, high intensity exercise is performed for a short time (usually $<1 \mathrm{~min}$ ) before a short rest to recover (usually $\leq 30 \mathrm{~s}$ ). After the rest period a new effort is made, and this cycle is repeated (see Buchheit and Laursen, 2013, for a review). Because of the short rest periods, there are few variations in the physiological responses to exercise, such as lung ventilation, heart rate and $\mathrm{O}_{2}$ uptake, despite large variations in the exercise intensity between exercise and recovery periods. In the Tabata protocol subjects cycle for $20 \mathrm{~s}$, rest for $10 \mathrm{~s}$ and continue this 20/10-cycle for $4 \mathrm{~min}$ (eight cycles) while the $\mathrm{O}_{2}$ uptake is measured continuously (Tabata et al., 1996, 1997). The exercise intensity in those studies was $170 \%$ of that corresponding to the maximal $\mathrm{O}_{2}$ uptake, which would normally exhaust the subject in $<1$ min if continued with no break. However, because of the short rest period, subjects are able to continue for $\approx 4$ min before reaching exhaustion.

It is well-established that high lung ventilation is aerobically demanding (Aaron et al., 1992; Dominelli et al., 2014), and consequently more than $10 \%$ of the cardiac output may be directed to the respiratory muscles (Harms et al., 1998). As a result, the $\mathrm{O}_{2}$ demand of the rest periods is expected to be considerably higher than at quiet rest with no prior highintensity exercise. If correctly performed, e.g., correcting for high ventilation during the rest periods, a short interval protocol may enable the examination of whether a linear extrapolation is justified for high-intensity exercise, such as treadmill running at high speeds or cross-country skiing on the treadmill, or whether there are significant non-linear effects. It may also allow for direct estimation of the rate of energy release without any extrapolation.

\section{Treadmill Exercise at Constant Speed}

As pointed out above, accelerations and decelerations of body segments may introduce non-linear effects. If exercise is performed on a treadmill at constant speed, that possible influence may be constant, independent of the physiological demand on the body.

\section{Mechanical Assistance}

For safety reasons subjects may wear a harness anchored to the ceiling during treadmill exercise. A separate rope attached to the harness may pull the subject forward, thus reducing the need for propulsive force developed by muscles. In that case the $\mathrm{O}_{2}$ demand of the exercise is expected to be reduced correspondingly. If the pulling force is recorded and in addition varied between experiments, a relationship between bodily developed propulsion force (or rope pulling force) and $\mathrm{O}_{2}$ demand may be established. The proposed design may also allow for measuring the propulsive force needed for treadmill exercise, for example during roller skiing, for comparison with theoretical estimates (Andersson and McGawley, 2018).

\section{Varying Treadmill Inclination}

Exercise on a level treadmill may be quite easy, while increasing the inclination will increase the physiological demand at constant speed. A relationship between treadmill inclination and steady state $\mathrm{O}_{2}$ uptake may be established for e.g., cycling on the treadmill or roller skiing and used for extrapolations if a linear relationship can be found. Our unpublished findings suggest that for running a corresponding relationship is not linear. The reason for the latter observation may be related to a vertical movement during the stride that varies with the inclination.

\section{CONCLUSIONS}

Relationships between exercise intensity and $\mathrm{O}_{2}$ demand vary between persons and should therefore be established individually. The computational method used for calculating the accumulated $\mathrm{O}_{2}$ deficit has considerable influence on the outcome. A protocol using $4 \mathrm{~min}$ stepwise increases in exercise intensity may enable the establishment of reliable relationships within $1 \mathrm{~h}$ of testing. That approach may be a suitable alternative to the reference method. Commonly used methods based on a fixed $y$-intercept (gross efficiency or net efficiency) are not recommended. Further methodological studies are needed, as proposed above.

\section{DATA AVAILABILITY STATEMENT}

The raw data supporting the conclusions of this article will be made available by the authors, without undue reservation.

\section{ETHICS STATEMENT}

Ethical review and approval was not required for the study on human participants in accordance with the local legislation and institutional requirements. The patients/participants provided their written informed consent to participate in this study.

\section{AUTHOR CONTRIBUTIONS}

JIM and BW developed the main idea of the study together. JIM conducted the experiments, analyzed the data, and drafted a manuscript. JIM and BW jointly revised the manuscript and approved the final version.

\section{ACKNOWLEDGMENTS}

We are most grateful to the subjects for their efforts during the experiments. We would like to thank the reviewers for their comments, which have improved the presentation, and further Paul Farmer for his excellent English editing. 


\section{REFERENCES}

Aaron, E., Johnson, B., Seow, C., and Dempsey, J. (1992). Oxygen cost of exercise hyperpnea: meas-urement. J. Appl. Physiol. 72, 1810-1817. doi: 10.1152/jappl.1992.72.5.1810

Andersson, E. P., and McGawley, K. (2018). A comparison between different methods of estimating anaerobic energy production. Front. Physiol. 9, 82. doi: 10.3389/fphys.2018.00082

Bickham, D., Le Rossignol, P., Gibbons, C., and Russell, A. P. (2002). Re-assessing accumulated oxygen deficit in middle-distance runners. J. Sci. Med. Sports 5, 372-382. doi: 10.1016/S1440-2440(02)80026-3

Boone, J., and Bourgois, J. (2012). The oxygen uptake response to incremental ramp exercise. Methodogical and physiological issues. Sports Med. 42, 511-526. doi: 10.2165/11599690-000000000-00000

Bosquet, L., Delhors, P. R., Duchene, A., Dupont, G., and Leger, L. (2007a). Anaerobic running capacity determined from a 3-parameter system model: relationship with other anaerobic indices and with running performance in the 800 m-run. Int. J. Sports Med. 28, 495-500. doi: 10.1055/s-2006-924516

Bosquet, L., Duchene, A., Dupont, G., Leger, L., and Carter, H. (2007b). $V \mathrm{O}_{2}$ kinetics during supramaximal exercise: relationship with oxygen deficit and 800-m running performance. Int. J. Sports Med. 28, 518-524. doi: 10.1055/s-2006-955896

Buchheit, M., and Laursen, P. B. (2013). High-intensity interval training, solutions to the programming puzzle: part I: cardiopulmonary emphasis. Sports Med. 43, 313-338. doi: 10.1007/s40279-013-0029-x

Buck, D., and McNaughton, L. (1999). Maximal accumulated oxygen deficit must be calculated using 10-min time periods. Med. Sci. Sports Exerc. 31, 1346-1349. doi: 10.1097/00005768-199909000-00018

Doherty, M. (1998). The effects of caffeine on the maximal accumulated oxygen deficit and short-term running performance. Int. J. Sport Nutr. 8, 95-104. doi: $10.1123 /$ ijsn.8.2.95

Doherty, M., Smith, P. M., Davison, R. C., and Hughes, M. G. (2002). Caffeine is ergogenic after supplementation of oral creatine monohydrate. Med. Sci. Sports Exerc. 34, 1785-1792. doi: 10.1097/00005768-200211000-00015

Doherty, M., Smith, P. M., and Schroder, K. (2000). Reproducibility of the maximum accumulated oxygen deficit and run time to exhaustion during short-distance running. J. Sports Sci. 18, 331-338. doi: 10.1080/026404100402395

Dominelli, P., Render, J., Molgat-Seon, Y., Foster, G., and Sheel, A. (2014). Precise mimicking of exercise hyperpnea to investigate the oxygen cost of breathing. Respir. Physiol. Neurobiol. 201, 15-23. doi: 10.1016/j.resp.2014. 06.010

Douglas, C. (1911). A method for determining the total respiratory exchange in man. J. Physiol. 42, 1-2.

Duffield, R., Edge, J., Bishop, D., and Goodman, C. (2007). The relationship between the $V \mathrm{O}_{2}$ slow component, muscle metabolites and performance during very-heavy exhaustive exercise. J. Sci. Med. Sport 10, 127-134. doi: 10.1016/j.jsams.2006.05.013

Ettema, G., and Lorås, H. W. (2009). Efficiency in cycling: a review. Eur. J. Appl. Physiol. 106, 1-14. doi: 10.1007/s00421-009-1008-7

Formenti, F., Minetti, A. E., and Borrani, F. (2015). Pedaling rate is an important determinant of human oxygen uptake during exercise on the cycle ergometer. Physiol. Rep. 3, 1-10. doi: 10.14814/phy2.12500

Gastin, P. B. (1994). Quantification of anaerobic capacity. Scand. J. Med. Sci. Sports 4, 91-112. doi: 10.1111/j.1600-0838.1994.tb 00411.x

Gastin, P. B. (2001). Energy system interaction and relative contribution during maximal exercise. Sports Med. 31, 725-741. doi: 10.2165/00007256-200131100-00003

Green, S., and Dawson, B. T. (1996). Methodological effects on the $\mathrm{VO}_{2}$-power regression and the accumulated $\mathrm{O}_{2}$ deficit. Med. Sci. Sports Exerc. 28, 392-397. doi: 10.1249/00005768-199603000-00016

Harms, C. A., Wetter, T. J., McClaran, S. R., Pegelow, D. F., Nickele, G. A., Nelson, W. B., et al. (1998). Effects of respiratory muscle work on cardiac output and its distribution during maximal exercise. J. Appl. Physiol. 85, 609-618. doi: 10.1152/jappl.1998.85.2.609

Hermansen, L. (1974). Oxygen transport during exercise in human subjects. Acta Physiol. Scand. 90, 1-104.
Hill, A. V., and Lupton, H. (1923). Muscular exercise, lactic acid, and the supply and utilization of oxygen. Q. J. Med. 16, 135-171. doi: 10.1093/qjmed/os-16.62.135

Krogh, A. (1913). A bicycle ergometer and respiration apparatus for the experimental study of muscular work. Scand. Arch. Physiol. 30, 375-394. doi: 10.1111/j.1748-1716.1913.tb00681.x

Mamen, A., Laparidist, C., and Van Den Tillaar, R. (2011). Precision in estimating maximal lactate steady state performance in running using a fixed blood lactate concentration or a delta value from an incremental lactate profile test. Int. J. Appl. Sports Sci. 23, 212-224. doi: 10.24985/ijass.2011.23.1.212

Maxwell, N. S., and Nimmo, M. A. (1996). Anaerobic capacity: a maximal anaerobic running test versus the maximal accumulated oxygen deficit. Can. J. Appl. Physiol. 21, 35-47. doi: 10.1139/h96-004

Medbø, J. I. (2002). Examination of the time response of the Metamax I and II metabolic analysers at the onset and end of exercise. Acta Kinesiol. Univ. Tartuensis 7, 50-60.

Medbø, J. I. (2010). "Accumulated oxygen deficit issues," in Exercise Physiology: from a Cellular to an Integrative Approach (Biomedical and Health Research), eds P. Connes, O. Hue, and S. Perrey (Amsterdam: IOS Press BV), 367-384.

Medbø, J. I., Gramvik, P., and Jebens, E. (1999). Aerobic and anaerobic energy release during $10 \mathrm{~s}$ and $30 \mathrm{~s}$ bicycle sprints. Acta Kinesiol. Univ. Tartuensis $4,122-146$.

Medbø, J. I., Mamen, A., Olsen, O. H., and Evertsen, F. (2000). Examination of four different instru-ments for measuring blood lactate concentration. Scand. J. Clin. Lab. Invest. 60, 367-379. doi: 10.1080/003655100750019279

Medbø, J. I., Mohn, A.-C., Tabata, I., Bahr, R., Vaage, O., and Sejersted, O. M. (1988). Anaerobic capacity determined by maximal accumulated O2 deficit. J. Appl. Physiol. 64, 50-60. doi: 10.1152/jappl.1988.64.1.50

Medbø, J. I., and Tabata, I. (1989). Relative importance of aerobic and anaerobic energy release during short-lasting exhausting bicycle exercise. J. Appl. Physiol. 67, 1881-1886. doi: 10.1152/jappl.1989.67.5.1881

Medbø, J. I., and Tabata, I. (1993). Anaerobic energy release in working muscle during $30 \mathrm{~s}$ to $3 \mathrm{~min}$ of exhausting bicycling. J. Appl. Physiol. 75, 1654-1660. doi: 10.1152/jappl.1993.75.4.1654

Nevill, A. M., Ramsbottom, R., Nevill, M. E., Newport, S., and Williams, C. (2008). The relative contributions of anaerobic and aerobic energy supply during track 100-, 400- and 800-m performance. J. Sports. Med. Phys. Fitness 48, 138-142.

Noordhof, D. A., de Koning, J. J., and Foster, C. (2010). The maximal accumulated oxygen deficit method. A valid and reliable measure of anaerobic capacity? Sports Med. 40, 285-302. doi: 10.2165/11530390-000000000-00000

Noordhof, D. A., Skiba, P. F., and de Koning, J. J. (2013). Determining anaerobic capacity in sporting activities. Int. J. Sports Physiol. Perform. 8, 475-482. doi: 10.1123/ijspp.8.5.475

Ramsbottom, R., Nevill, A. M., Nevill, M. E., Newport, S., and Williams, C. (1994). Accumulated oxygen deficit and short-distance running performance. J. Sports Sci. 12, 447-453. doi: 10.1080/02640419408732194

Ramsbottom, R., Nevill, A. M., Seager, R. D., and Hazeldine, R. (2001). Effect of training on accumulated oxygen deficit and shuttle run performance. J. Sports Med. Phys. Fitness 41, 281-290.

Ramsbottom, R., Nevill, M. E., Nevill, A. M., and Hazeldine, R. (1997). Accumulated oxygen deficit and shuttle run performance in physically active men and women. J. Sports Sci. 15, 207-214. doi: 10.1080/0264041973 67489

Riojas, A. E., Valenzuela, J. R., McFarlin, B. K., Vingren, J. L., and Hill, D. W. (2020). The increase in oxygen demand during severe intensity exercise must be included in calculation of oxygen deficit. Int. J. Exercise Sci. 13, 645-655.

Russell, A. P., Le Rossignol, P., and Lo, S. K. (2000). The precision of estimating the total energy demand: implications for the determination of the accumulated oxygen deficit. J. Exerc. Physiol. 3, 55-63.

Scott, C. B., Roby, F. B., Lohman, T. G., and Bunt, J. C. (1991). The maximally accumulated oxygen deficit as an indicator of anaerobic capacity. Med. Sci. Sports Exerc. 23, 618-624. doi: 10.1249/00005768-199105000 00015

Tabata, I., Irisawa, K., Kouzaki, M., Nishimura, K., Ogita, F., and Miyachi, M. (1997). Metabolic profile of high intensity intermittent exercises. Med. Sci. Sports Exerc. 29, 390-395. doi: 10.1097/00005768-19970300000015 
Tabata, I., Nishimura, K., Kouzaki, M., Hirai, Y., Ogita, F., Miyachi, M., et al. (1996). Effects of moderate-intensity endurance and high-intensity intermittent training on anaerobic capacity and VO2max. Med. Sci. Sports Exerc. 28, 1327-1330. doi: 10.1097/00005768-199610000-00018

Taylor, H. L., Buskirk, E., and Henschel, A. (1955). Maximal oxygen intake as an objective measure of cardio-respiratory performance. J. Appl. Physiol. 8, 73-80. doi: 10.1152/jappl.1955.8.1.73

Wadley, G., and le Rossignol, P. (1998). The relationship between repeated sprint ability and the aerobic and anaerobic energy systems. J. Sci. Med. Sport 1, 100-110. doi: 10.1016/S1440-2440(98)8 0018-2

Whipp, B. J., and Wasserman, K. (1986). Effect of anaerobiosis on the kinetics of $\mathrm{O}_{2}$ uptake during exercise. Fed. Proc. 45, 2942-2947.

Zoladz, J. A., Duda, K., and Majerczak, J. (1998). $\mathrm{VO}_{2} /$ power output relationship and the slow component of oxygen uptake kinetics during cycling at different pedalling rates: relationship to venous lactate accumulation and blood lactate acid-base balance. Physiol. Res. 47, 428-438.
Conflict of Interest: The authors declare that the research was conducted in the absence of any commercial or financial relationships that could be construed as a potential conflict of interest.

Publisher's Note: All claims expressed in this article are solely those of the authors and do not necessarily represent those of their affiliated organizations, or those of the publisher, the editors and the reviewers. Any product that may be evaluated in this article, or claim that may be made by its manufacturer, is not guaranteed or endorsed by the publisher.

Copyright $\odot 2022$ Medbø and Welde. This is an open-access article distributed under the terms of the Creative Commons Attribution License (CC BY). The use, distribution or reproduction in other forums is permitted, provided the original author(s) and the copyright owner(s) are credited and that the original publication in this journal is cited, in accordance with accepted academic practice. No use, distribution or reproduction is permitted which does not comply with these terms. 